\title{
Intrasession and Intersession Reliability of Isometric Trunk and Hip Strength Measurements Using the Portable Tension Dynamometer
}

\author{
Sakiko Oyama, Edgar Garza, and Kylie Dugan
}

\begin{abstract}
Context: The trunk/pelvis is an important link between the upper- and lower-extremities. Therefore, assessing strength of the trunk and hip muscles that control the segments is clinically meaningful. While an isokinetic dynamometer can be used to measure trunk strength, the equipment is expensive and not portable. Objective: To test the reliability of simple trunk and hip strength measures that utilize a bar, straps, and a portable tension dynamometer. Design: Test-retest reliability study. Setting: Biomechanics research laboratory. Patients (or Other Participants): Twenty college-age individuals (10 males/10 females, age $=20.9$ [3.7] y) participated. Intervention(s): The participants attended 2 testing sessions, 1 week apart. The participants' trunk-flexion, rotation, and hip abduction strength were measured at each session. Main Outcome Measures: Peak trunk flexion, rotation, and hip abduction forces were normalized to the participant's body weight (BW). In addition, hip-abduction torque was calculated by multiplying the force times the leg length and normalized to BW. The trial data from both sessions were used to calculate the intrasession reliability, and the averages from the 2 sessions were used to calculate the intersession reliability. Intraclass correlation coefficients, SEM, and minimal detectable change were calculated to evaluate reliability of measures. Results: The intrasession intraclass correlation coefficients (SEM) for trunk flexion, rotation, hip abduction, and hip abduction torque were $.837(5.2 \% \mathrm{BW}), .978(1.3 \% \mathrm{BW}), .955(1.0 \% \mathrm{BW})$, and $.969(5.8 \mathrm{~N} \cdot \mathrm{m} / \mathrm{BW})$, respectively. The intersession reliability for trunk flexion, rotation, hip abduction, and hip abduction torque were $.871(4.3 \% \mathrm{BW}), .801(3.8 \% \mathrm{BW}), .894(1.5 \%$ BW), and $.968(5.9 \mathrm{~N} \cdot \mathrm{m} / \mathrm{BW})$, respectively. Conclusions: The measures of trunk and hip abduction strength are highly repeatable within a session. The reliability of the measures between sessions was also good/excellent with relatively small SEM and minimal detectable change. The tests described in this study can be used to assess changes in trunk/hip strength over time.
\end{abstract}

Keywords: trunk strength, rotation, isometric trunk strength

Trunk and hip muscles play a critical role in controlling the motion of the trunk-pelvis segment and transferring energy between the lower- and upper-extremities. ${ }^{1}$ Therefore, it is important to establish a reliable method to measure trunk and hip muscle strength that is also accessible. Isokinetic dynamometers, ${ }^{2-4}$ handheld dynamometers (HHDs), ${ }^{5}$ and functional/clinical tests, ${ }^{2,6}$ such as the Sorensen test and plank test, are commonly used to assess trunk muscle strength and endurance. The isokinetic dynamometer allows us to evaluate trunk strength in all 3 planes throughout the range of motion at various movement speeds and testing modes (ie, concentric, isometric, eccentric modes). ${ }^{3,4}$ However, they are expensive and not portable. HHDs are cost-effective and portable equipment that can be used to assess strength. Good reliability has been reported for the assessment of trunk flexion using the HHD. ${ }^{5}$ However, HHDs have not been used to assess trunk rotation strength, likely because there is no good placement for HHDs that provides leverage and stability when assessing trunk rotation strength. Functional tests can be administered without any specialized equipment. However, they are not intended to measure the strength of a specific muscle group.

To overcome the limitations of the existing methods to evaluate trunk strength, we have devised a method to assess trunk strength using relatively inexpensive and portable pieces of equipment. While the method was primarily developed to assess trunk rotation strength, it can also measure trunk flexion and hip

The authors are with the Applied Biomechanics Research Laboratory, The University of Texas at San Antonio, San Antonio, TX, USA. Oyama (sakiko.oyama@utsa. edu) is corresponding author. abduction strength. The purpose of the study was to test the intrasession and intersession reliability of the simple method of assessing trunk flexion and rotation strength and hip abduction strength that utilizes a bar, straps, and a portable tension dynamometer.

\section{Methods}

A total of 20 college-aged individuals who were free of any musculoskeletal injuries participated in this study (10 males/10 females, age $=20.9$ [3.7] $\mathrm{y}$, height $=1.68[0.10] \mathrm{m}$, mass $=67.8[10.9] \mathrm{kg}$ ). All participants provided informed consent before participating in the study. The participants attended 2 sessions in the research laboratory scheduled approximately 1 week apart. During each session, participants went through the measurement of trunk flexion, trunk rotation, and hip abduction strength (Figures 1-3).

For the trunk strength tests, we constructed an extension to the portable treatment table that allowed the participant to rest their head and upper back (Figures 1 and 2). The dynamometer (DMG500 Muscle Strength Evaluator; AMETEK Inc, Berwyn, PA) was secured vertically onto a plank that was placed on the floor, in line with the treatment table. The plank with the dynamometer was able to move side to side under the second plank, which was held down by the legs of the treatment table and the participant's body weight.

For trunk strength tests, the participants lay supine on a treatment table with their head and upper torso resting on the table extension, hips flexed $45^{\circ}$ and knees flexed $90^{\circ}$. The participants were guided to slide up and/or down on the table until the line connecting the axillae came directly above the dynamometer. A bar 
made of solid wood was placed over the participant's chest at armpit level. Participants crossed their forearms across the chest to hold the bar in place. The straps were attached to the bar using velcro strap to prevent sliding. The straps were adjusted so that they hung just lateral to the armpit. The straps were connected to the dynamometer using a carabiner, and the length was adjusted so that they were taut when participants were at rest. Straps on both sides of the trunk were used to test trunk-flexion strength. The strap on one side was used for the trunk rotation strength test.

Trunk-flexion strength was measured as the peak force recorded by the dynamometer as the participants attempted to lift their torso as if performing a crunch (Figure 1). The participants pulled against the unyielding straps with a maximum effort. Trunk rotation strength was measured as the participants attempted to lift their shoulder toward the contralateral hip as if performing an oblique crunch (Figure 2). The participants pulled against the unyielding strap on the side of the shoulder that they attempted to lift with maximum effort. For example, when the participant lifted his/her right shoulder to rotate the trunk to the left, the participant pulled against the strap on the right side. Trunk rotation strength was measured in both directions.

Hip abduction strength was measured on the right side for all participants. The hip abduction strength was measured with participants lying on their left side with the right leg (ie, testing limb) extended and the left knee flexed $90^{\circ}$ (Figure 3). The participants were guided to slide up and/or down on the table until the right ankle was positioned directly above the dynamometer. A strap was attached to the velcro strap that was wrapped around the ankle and was adjusted to become taut when the testing limb was horizontal. The hip abduction strength was measured as the participants abducted their hip against the strap. The measurements were taken 3 times, or until 2 consistent $( \pm 0.45 \mathrm{~kg})$ values were recorded.

The trunk rotation strength to the right and left were pooled before the data analysis. The strength was calculated as the force normalized to the participant's body weight. In addition, the hip abduction torque was calculated by multiplying the force by the leg length estimated from the subject's height (53.0\% of the height ${ }^{7}$ ) and normalized to body weight. The first 2 measures from both sessions were used to calculate the intrasession reliability, and the averages from the 2 sessions were used to calculate the intersession reliability. The reliability was evaluated through calculation of the intraclass correlation coefficients $\left(\mathrm{ICC}_{2, k}\right)$ and the $\mathrm{SEM}=\mathrm{SD} \times \sqrt{1-\mathrm{ICC}}$. The ICC values below between .5 and .75 indicate moderate reliability, values between .75 and .9 indicate good reliability, and values greater than .90 indicate excellent reliability. ${ }^{8}$ In addition, the minimal detectable changes (MDCs) were calculated as SEM $\times$ $1.96 \times \sqrt{2}$.

\section{Results}

The intrasession and intersession reliability (ICC and SEM) and MDCs are reported in Table 1.

\section{Discussion}

The purpose of the study was to test the intrasession and intersession reliability of the simple method of assessing trunk flexion and rotation strength and hip abduction strength that utilizes a bar, straps, and a portable tension dynamometer. The intrasession reliability was good (>.75) for the trunk-flexion strength and excellent (>.9) for the trunk rotation strength and hip abduction

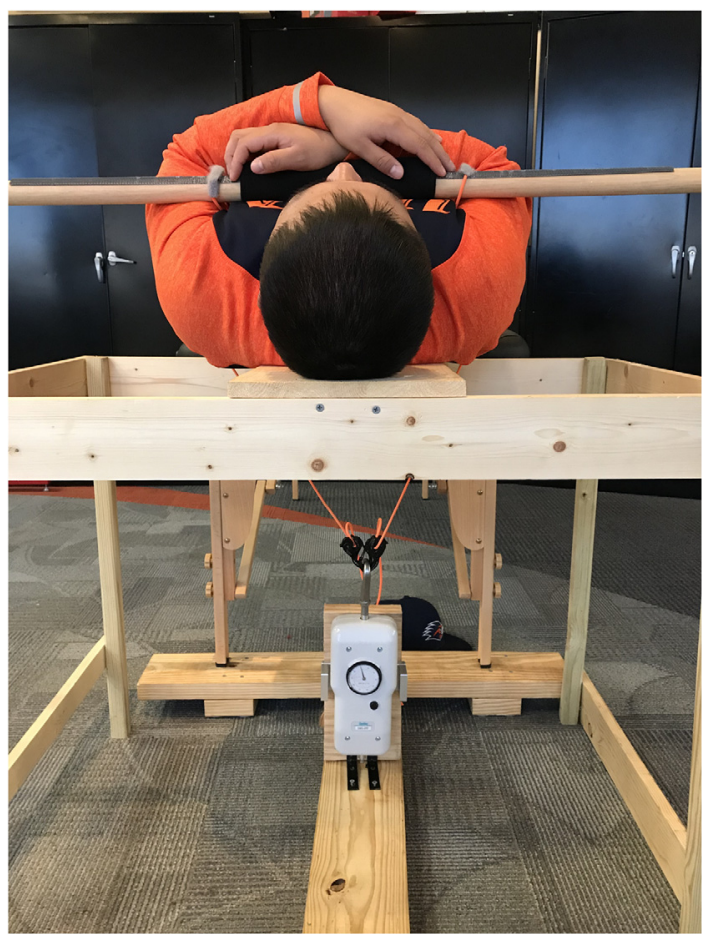

Figure 1 - Assessment of trunk-flexion strength.

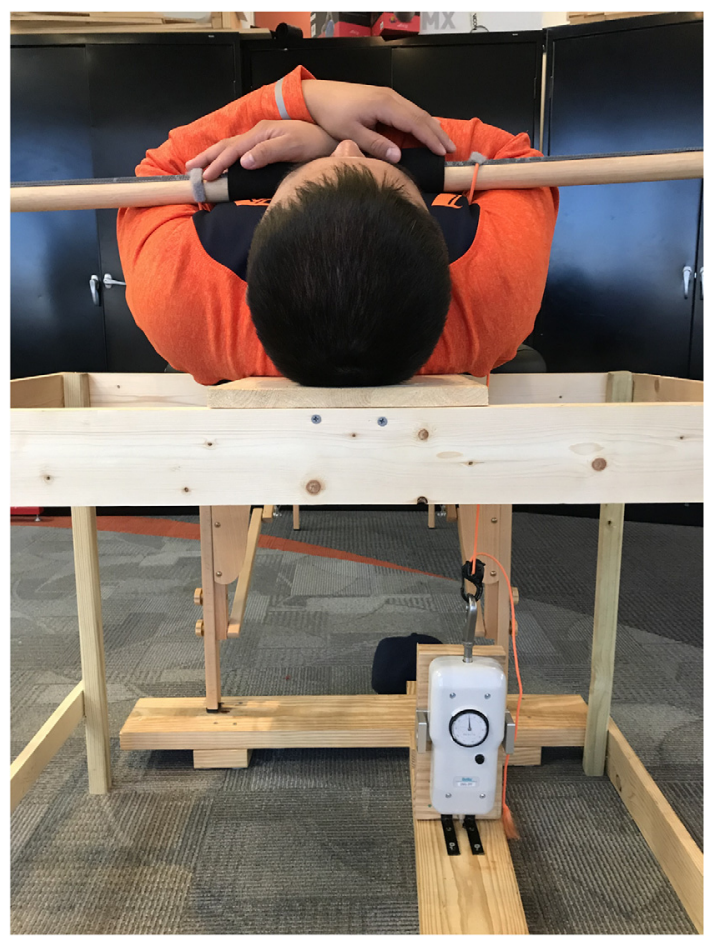

Figure 2 - Assessment of trunk rotation strength.

strength, indicating that these measures are highly repeatable within a session. The reliability of the measures between sessions was also good for the trunk strength measures and excellent (>.9) for the hip abduction strength, with relatively small SEM and MDC 


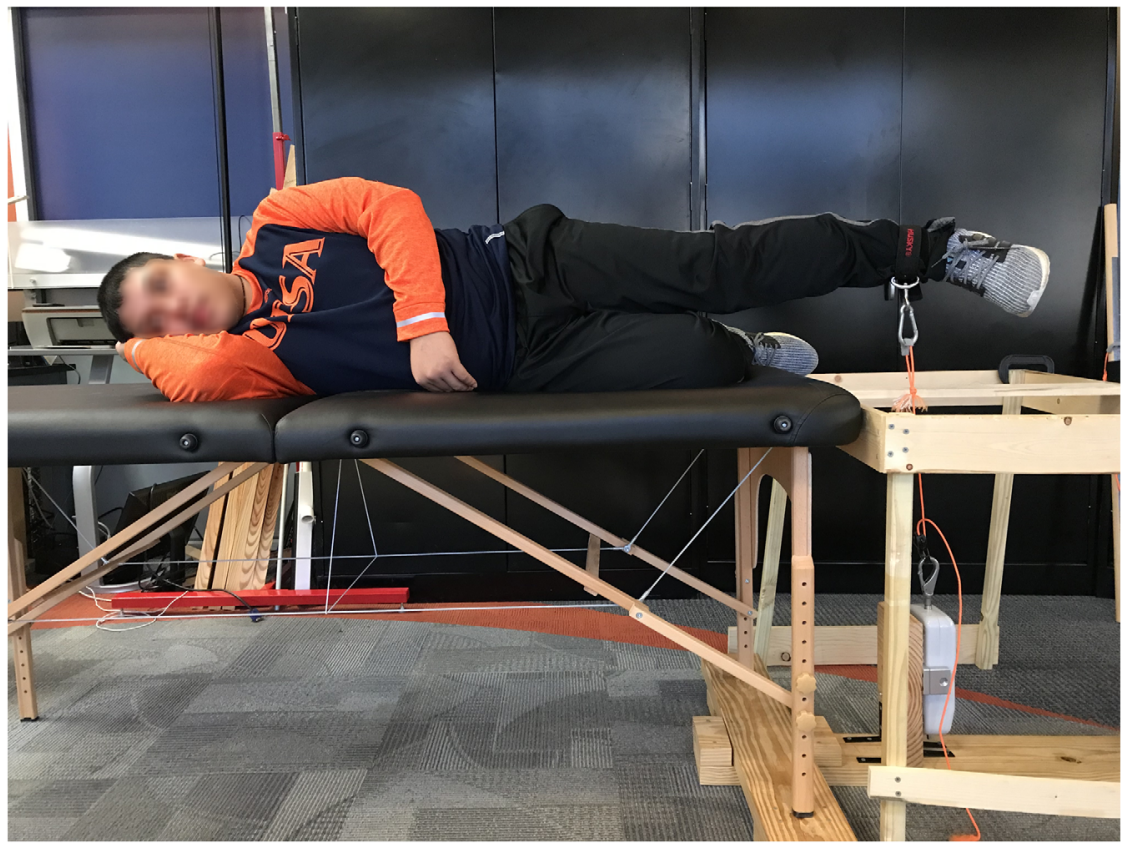

Figure 3 - Assessment of hip abduction strength.

Table 1 Intrasession and Intersession Reliability of the Measurements

\begin{tabular}{|c|c|c|c|c|c|}
\hline \multirow[b]{2}{*}{ Strength variables } & \multicolumn{2}{|c|}{$\begin{array}{c}\text { Intrasession } \\
\text { reliability }\end{array}$} & \multicolumn{2}{|c|}{$\begin{array}{c}\text { Intersession } \\
\text { reliability }\end{array}$} & \multirow{2}{*}{$\begin{array}{l}\text { Minimal } \\
\text { detectable } \\
\text { change }\end{array}$} \\
\hline & ICC & SEM & ICC & SEM & \\
\hline Trunk flexion, \%BW & .837 & 5.2 & .871 & 4.3 & 11.9 \\
\hline Trunk rotation, \%BW & .978 & 1.3 & .801 & 4.3 & 11.9 \\
\hline Hip abduction, \%BW & .955 & 1.0 & .894 & 1.5 & 4.1 \\
\hline Hip abduction torque, $\mathrm{N} \cdot \mathrm{m} / \mathrm{BW}$ & .969 & 5.8 & .968 & 5.9 & 16.3 \\
\hline
\end{tabular}

Abbreviations: \%BW, percentage of body weight; ICC, intraclass correlation coefficient.

values. The advantage of the method presented in this study is that it is able to assess trunk rotation strength without the use of an isokinetic dynamometer. The intersession reliability of the trunk rotation strength using isokinetic dynamometers is good, especially in the isometric testing mode and at lower movement velocities. ${ }^{3,9}$ In one study, the ICCs for trunk rotation strength during isometric, isokinetic at $60 \%$, and isokinetic at $150 \%$ s ranged from .82 to $.93 .^{3}$ The reliability of our method ( $\mathrm{ICC}=.80$ ) was somewhat lower than these reported values, yet is still considered good $(>.75)$ and has relatively small SEM and MDC values. Trunk rotation kinematics, which would depend on the forces generated by the trunk rotators, has been linked to performance in sports that require rapid trunk rotation (eg, golf swing and baseball pitching). ${ }^{1}$ However, there is a limited amount of research that measures trunk rotation strength or examines the association between the trunk rotation strength and injury risk and/or performance. The lack of studies, at least in part, is attributed to the limited availability of the isokinetic dynamometers with specialized attachments. Having a reliable method to assess trunk rotation strength may allow more studies to be conducted in this area.

The reliability of the trunk flexion and hip abduction strength was also comparable with what was reported for the previously described method using the HHD, ${ }^{5}$ indicating that they can be assessed using the same setup as that used for the trunk rotation strength. Decreased trunk-flexion strength has been associated with low back pain and increased fall risk in older adults, ${ }^{10}$ and decreased hip abduction strength has been linked to alterations in the frontal plane trunk, pelvis, and lower-extremity kinematics, which can lead to a variety of lower-extremity injuries.

In conclusion, a simple, portable, and cost-effective method of measuring trunk flexion and rotation and hip abduction strength presented in this study has high intrasession and intersession reliability. When the method is used in future studies, the SEM and MDC values reported in this study can be used to evaluate the magnitude of differences or changes in trunk and hip strength measured using the method.

\section{References}

1. Stodden DF, Fleisig GS, McLean SP, Andrews JR. Relationship of biomechanical factors to baseball pitching velocity: within pitcher variation. J Appl Biomech. 2005;21(1):44-56. PubMed ID: 16131704 doi:10.1123/jab.21.1.44 
2. Juan-Recio C, Lopez-Plaza D, Barbado Murillo D, Garcia-Vaquero MP, Vera-Garcia FJ. Reliability assessment and correlation analysis of 3 protocols to measure trunk muscle strength and endurance. $J$ Sports Sci. 2018;36(4):357-364. PubMed ID: 28357922 doi:10. 1080/02640414.2017.1307439

3. Roth R, Donath L, Kurz E, Zahner L, Faude O. Absolute and relative reliability of isokinetic and isometric trunk strength testing using the IsoMed-2000 dynamometer. Phys Ther Sport. 2017;24:26-31. PubMed ID: 27964928 doi:10.1016/j.ptsp.2016.11.005

4. Zouita AB, Zouita S, Ben Salah FZ, Benm DG, Chaouachi A. Isokinetic trunk strength, validity, reliability, normative data and relation to physical performance and low back pain: a review of the literature. Int J Sports Phys Ther. 2020;15(1):160-174. doi:10. 26603/ijspt20200160

5. De Blaiser C, De Ridder R, Willems T, Danneels L, Roosen P. Reliability and validity of trunk flexor and trunk extensor strength measurements using handheld dynamometry in a healthy athletic population. Phys Ther Sport. 2018;34:180-186. PubMed ID: 30366246 doi:10.1016/j.ptsp.2018.10.005

6. McGill SM, Childs A, Liebenson C. Endurance times for low back stabilization exercises: clinical targets for testing and training from a normal database. Arch Phys Med Rehabil. 1999;80(8):941-944. PubMed ID: 10453772 doi:10.1016/S0003-9993(99)90087-4

7. Drillis R, Contini R, Bluestein M. Body segment parameters. Artif Limbs. 1964;8(1):44-66.

8. Portney LG, Watkins MP. Foundations of Clinical Research: Applications to Practice. Upper Saddle River, NJ: Pearson Education Inc; 2009.

9. Lindsay DM, Horton JF. Trunk rotation strength and endurance in healthy normals and elite male golfers with and without low back pain. N Am J Sports Phys Ther. 2006;1(2):80-89. PubMed ID: 21522218

10. Kato S, Murakami H, Demura S, et al. Abdominal trunk muscle weakness and its association with chronic low back pain and risk of falling in older women. BMC Musculoskelet Disord. 2019;20(1):273. PubMed ID: 31159812 doi:10.1186/s12891-019-2655-4 